\title{
O USO DA ESTIMULAÇÃO MAGNÉTICA TRANSCRANIANA DE BAIXA FREQUÊNCIA NO TRATAMENTO DA DEPRESSÃO NO HOSPITAL UNIVERSITÁRIO DE BRASÍLIA
}

\section{Achados preliminares}

\author{
Joaquim P. Brasil-Neto1, Raphael Boechat-Barros², Doralúcia A. da Mota-Silveira ${ }^{3}$
}

\begin{abstract}
RESUMO - O presente estudo relata o uso da estimulação magnética transcraniana de baixa frequência sobre o córtex pré-frontal direito em três pacientes com diagnóstico de episódio depressivo maior, de acordo com o DSM - IV. Houve melhora significativa em dois pacientes, com diminuição de mais de $50 \%$ na pontuação da escala de Hamilton - 17 itens. São feitas considerações acerca de possíveis indicações e limitações do seu uso clínico, bem como sugestões ao protocolo de uso desta técnica. Também são discutidos fatores socioeconômicos relacionados a esta terapia.
\end{abstract}

PALAVRAS-CHAVE: estimulação magnética transcraniana, baixa frequência, depressão.

\begin{abstract}
The use of slow-frequency transcranial magnetic stimulation in the treatment of depression at Brasília University Hospital: preliminary findings

ABSTRACT - This paper reports the use of slow frequency transcranial magnetic stimulation of the right prefrontal cortex in three patients with a diagnosis of major depressive episode according to the DSM-IV classification. There was a significant improvement in two patients, with a decrease of over $50 \%$ in the Hamilton Scale scores- 17 items. Possible indications and limitations of this therapeutic tool are discussed, as well as socio-economic aspects of this new treatment.
\end{abstract}

KEY WORDS: transcranial magnetic stimulation, slow frequency, depression.

A estimulação magnética transcraniana (EMT) foi introduzida por Barker et al. em 1985', mostrandose útil para o estudo das vias motoras e o mapeamento topográfico não-invasivo do córtex motor humano ${ }^{2-4}$. A técnica utiliza um aparelho capaz de produzir um campo eletromagnético, usualmente da ordem de 2 tesla (40 000 vezes o campo magnético da terra e aproximadamente da mesma intensidade do campo magnético estático produzido por um aparelho de ressonância magnética), o qual é conduzido através de uma bobina que entra em contato com o couro cabeludo do indivíduo. Este campo eletromagnético atravessa o crânio estimulando uma área cortical próxima, através da indução de cargas elétricas no parênquima cerebral (indução eletro magnética - lei de Faraday). A princípio trata-se de uma forma de estimulação elétrica sem eletrodos, não havendo necessidade de craniotomia. Utilizando-se bobina em forma de oito obtêm-se resultados que se relacionam bem com aqueles produzidos pela estimulação cortical elétrica direta.

Inicialmente utilizada na propedêutica com intuito de pesquisar alterações das vias motoras, a EMT passou a ser utilizada também como forma terapêutica em patologias como doença de Parkinson ${ }^{5}$ e epilepsia ${ }^{6}$ e, há cerca de 6 anos, foram iniciadas pesquisas no tratamento da depressão ${ }^{7}$, com grande variedade de resultados ${ }^{8}$.

Laboratório de Neurobiologia, Departamento de Ciências Fisiológicas, Universidade de Brasília (UNB), Brasília-DF, Brasil; ${ }^{1}$ Neurologista, Doutor em Ciências pela Universidade Federal do Rio de Janeiro, Coordenador da Disciplina Neurofisiologia Médica da UNB; ${ }^{2}$ Psiquiatra, mestrando da Faculdade de Ciências da Saúde da UNB; ${ }^{3}$ Fisioterapeuta, mestranda da Faculdade de Ciências da Saúde da UNB.

Recebido 2 Maio 2002, recebido na forma final 17 Julho 2002. Aceito 7 Agosto 2002.

Dr. Joaquim P. Brasil-Neto - Laboratorio de Neurologia, Departamento de Ciências Fisiológicas, ICC Sul, Módulo 8 - UNP - Asa Norte 70910-900 Brasília DF - Brasil. 
Em relação ao número de pulsos por unidade de tempo, existem dois tipos de EMT: baixa frequência $=1 \mathrm{~Hz}$ e alta frequência $>1 \mathrm{~Hz}$, com efeitos diversos. $\mathrm{O}$ uso da estimulação magnética de alta frequência aumenta o fluxo sanguíneo cerebral na área, medido através de PET (positron emissiom tomography), com consequente aumento da atividade cerebral. A estimulação de baixa frequência, por outro lado, diminui a atividade cerebral ${ }^{9}$. Menkes et al. ${ }^{10}$ sugeriram que a depressão maior deve ser o resultado de uma diminuição da função do lobo frontal esquerdo em relação ao direito. Baseados nesta hipótese, propuseram o tratamento com a estimulação magnética transcraniana de baixa frequência sobre o córtex frontal direito, com intuito de diminuir a atividade naquela área. Atualmente, o córtex pré-frontal dorsolateral é o principal alvo dos estudos envolvendo a EMT no tratamento da depressão, que estaria ligada à regulação de conexão de regiões cerebrais (incluindo as regiões corticais pré-frontal, parietal, temporal e cíngulo, bem como partes do estriado, tálamo e hipotálamo) ${ }^{8}$.

O presente estudo descreve os resultados preliminares obtidos durante o projeto de implantação do uso da estimulação magnética transcraniana de baixa frequência como tratamento para depressão no Hospital Universitário de Brasília.

\section{MÉTODO}

Neste estudo utilizamos um aparelho Dantec ${ }^{\circledR}$ Maglite, o qual teve o seu uso aprovado pela agência de saúde norte americana FDA (Food and Drug Administration), em 1993, sob o registro - K931923. Este estudo foi aprovado pelo Comitê de Ética em Pesquisa - CEP/FS - da Faculdade de Ciências da Saúde da UNB, seguindo as diretrizes da resolução 196/96 do Conselho Nacional de Saúde /Minis- tério da Saúde. Os pacientes, antes de participarem do estudo, assinaram um termo de consentimento livre e esclarecido, em que estava descrito o mecanismo de ação da EMT, bem como os possíveis efeitos colaterais e contra-indicações.

Foram estudados três pacientes com diagnóstico de episódio depressivo maior segundo o DSM - IV (Manual Diagnóstico e Estatístico da Associação Americana de Psiquiatria) ${ }^{11}$, considerados de difícil tratamento por seus psiquiatras clínicos, seja devido à não resposta ou à intolerância medicamentosa. As Pacientes 1 e 2 apresentavam sintomas psicóticos associados (Tabela 1) mas, por diferentes motivos, não estavam em uso de neurolépticos durante a pesquisa: a primeira, por estar em investigação clínica dos sintomas motores que apresentava, sendo posteriormente diagnosticada discinesia tardia devido ao uso prévio desta medicação: a segunda, por ter diagnóstico muito recente de sintomas psicóticos, não tendo ainda iniciado o uso de medicação específica. Nestas pacientes foi aplicada a escala Hamilton de 17 itens ${ }^{12}$ em três momentos: T1 - antes da primeira aplicação, T2 - na metade do estudo e T3 - no final do mesmo, com o objetivo de quantificar uma possível melhora.

As medicações foram mantidas nas mesmas dosagens de antes do início do tratamento durante todo o protocolo da pesquisa, com o intuito de evitar viés de melhora ou piora clínica (Tabela 1).

Em cada paciente foram aplicadas 8 sessões de estimulação magnética transcraniana de baixa frequência - 0,5 Hz - sendo 2 por semana, cada uma com 5 séries de 20 estímulos com intervalo de 1 minuto entre cada série, aplicados sobre o córtex pré-frontal dorsolateral direito, $5 \mathrm{~cm}$ à frente do ponto ótimo para estimular o primeiro interósseo dorsal ${ }^{13}$.

Para se calcular a intensidade do estímulo usamos como referência o limiar motor, que é a intensidade mínima de estímulo capaz de produzir movimentos visíveis da musculatura da mão contralateral em pelo menos 3 de 5

Tabela 1. Pacientes estudados.

\begin{tabular}{|c|c|c|c|c|c|c|c|}
\hline Paciente & $\begin{array}{l}\text { Idade } \\
\text { (anos) }\end{array}$ & Sexo & $\begin{array}{c}\text { Tempo de tratamento } \\
\text { tratamento para } \\
\text { depressão (meses) }\end{array}$ & $\begin{array}{l}\text { Medicação } \\
\text { em uso }\end{array}$ & $\begin{array}{l}\text { Uso prévio de } \\
\text { eletroconvulsoterapia }\end{array}$ & Comorbidades & Diagnóstico \\
\hline 1 & 54 & $\mathrm{~F}$ & 24 & $\begin{array}{l}\text { Fluoxetina } \\
20 \text { mg/dia e } \\
\text { diazepan } \\
10 \text { mg/dia }\end{array}$ & Não & $\begin{array}{l}\text { Discinesia } \\
\text { Tardia }\end{array}$ & $\begin{array}{l}\text { Depressão } \\
\text { com } \\
\text { sintomas } \\
\text { psicóticos }\end{array}$ \\
\hline 2 & 30 & $\mathrm{~F}$ & 7 & $\begin{array}{l}\text { Paroxetina } \\
30 \mathrm{mg} / \mathrm{dia} \text { e } \\
\text { clonazepan } \\
2,0 \mathrm{mg} / \mathrm{dia}\end{array}$ & Não & Nega & $\begin{array}{l}\text { Depressão } \\
\text { com } \\
\text { sintomas } \\
\text { psicóticos }\end{array}$ \\
\hline 3 & 31 & $\mathrm{~F}$ & 3 & $\begin{array}{l}\text { Venlafaxina } \\
75 \text { mg/dia }\end{array}$ & Não & Nega & Depressão \\
\hline
\end{tabular}


pulsos simples aplicados sobre o córtex motor. Este método de quantificação do limiar motor foi escolhido em vez da eletromiografia porque, na maioria dos serviços de psiquiatria, não existe eletroneuromiógrafo e a sua aquisição geraria custo igual ou superior ao do aparelho de EMT, além de aumentar a complexidade do procedimento. Não se pode excluir que exista alguma discrepância quanto ao local exato de estimulação dos nossos pacientes se comparados aos de Pascual-Leone et al. ${ }^{13}$, em virtude da utilização de critérios diferentes para a determinação do ponto ótimo da estimulação do primeiro interósseo dorsal e consequente limiar motor (critério clínico versus eletromiógráfico). Entretanto, pequenos deslocamentos da bobina não afetam significativamente as características da estimulação magnética dado o caráter pouco focal da mesma, quando comparada à estimulação elétrica ${ }^{14}$. Como também relatado por Pascual-Leone et al. ${ }^{13}$, a estimulação na área descrita por sua técnica não estimula apenas a área 46 de Brodmann, mas também a área 9, indicando relativa falta de precisão. Neste estudo foram usados estímulos a $100 \%$ do limiar motor.

\section{RESULTADOS}

O limiar motor médio das Pacientes 1 e 2 foi 33,12\% da potência máxima do aparelho, e da Paciente 3, 40\%.

A Paciente 1 apresentava quadro de depressão psicótica grave (38 pontos), não se observando meIhora significativa.
A Paciente 2 também apresentava sintomas psicóticos, igualmente classificada como grave (34 pontos). Se, por um lado, esta paciente teve melhora clínica que pode ser considerada significativa (diminuição de mais de $50 \%$ na pontuação de HAMD), por outro lado sua pontuação ao final da pesquisa ainda configurava depressão leve.

A Paciente 3 não apresentava sintomas psicóticos e sua depressão era classificada como moderada (22 pontos). Apresentou grande melhora com o tratamento, terminando com 2 pontos.

\section{DISCUSSÃO}

Neste estudo observamos perfis de resposta diferentes ao mesmo tratamento. A paciente que teve a maior melhora foi a que apresentava a menor pontuação na escala e sem sintomas psicóticos. Chamou a atenção o fato de não haver melhora em nenhum dos sintomas psicóticos, inclusive alucinações auditivas, presentes em um dos pacientes, ao contrário do que demonstraram alguns estudos envolvendo pacientes com este sintoma ${ }^{15,16}$. A diminuição contínua da pontuação de todos os pacientes ao longo do estudo pode sugerir que maior número de sessões, com o prolongamento do tratamento

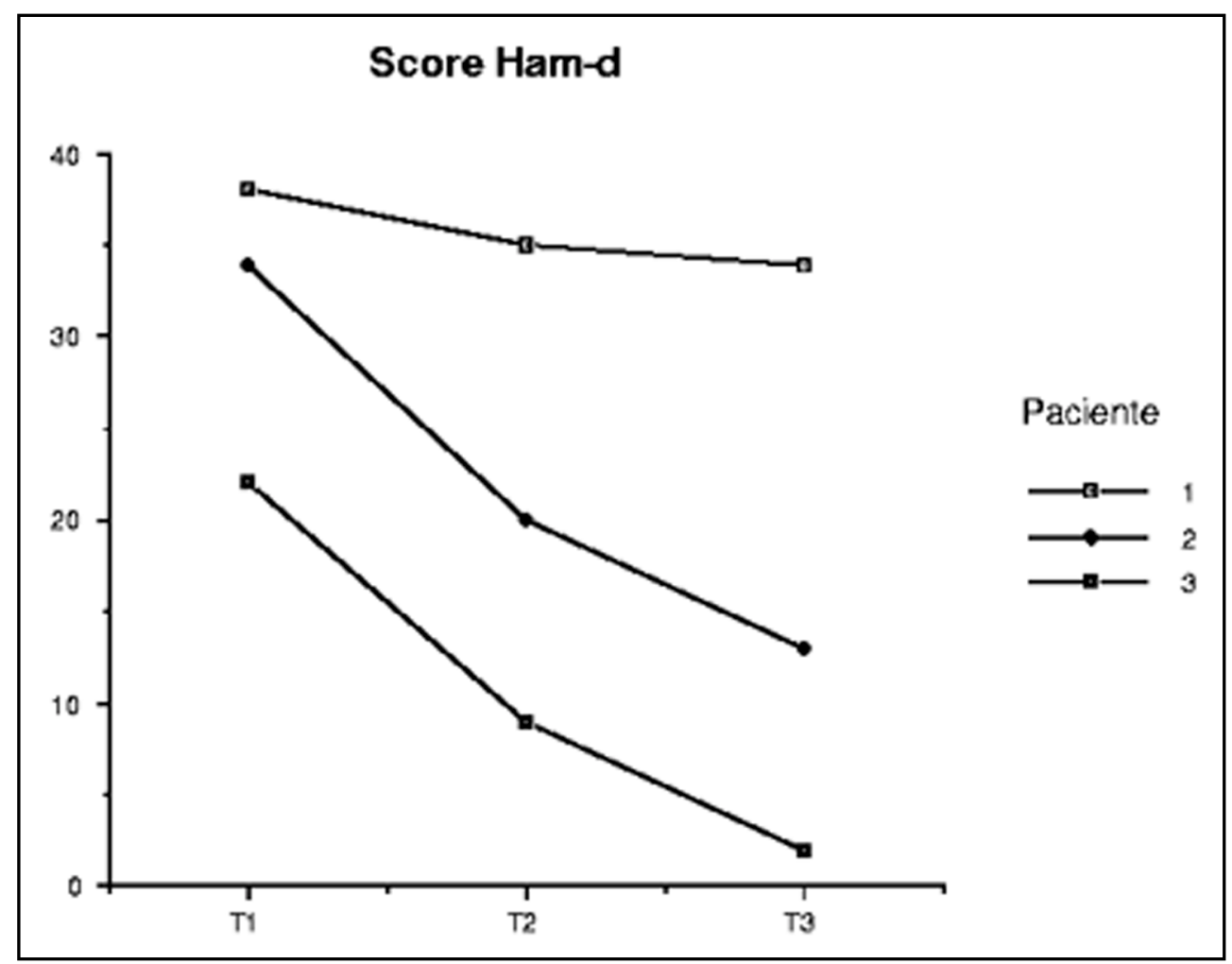

Fig 1. Evolução da pontuação na escala de Hamilton nos três pacientes; T1 - início do tratamento, $T 2$ metade do tratamento, $T 3$ - final do tratamento. Paciente $1: T 1=38$ pts, $T 2=35$ pts, $T 3=34$ pts . Paciente $2: T 1=34$ pts, $T 2=20 p t s, T 3=13 p t s$. Paciente $3: T 1=22$ pts, $T 2=9$ pts, $T 3=2$ pts. 
(mais do que quatro semanas) leve a melhora mais significativa do respectivo quadro clínico.

A estimulação magnética transcraniana de baixa frequência apresenta pelo menos duas grandes vantagens em relação à de alta frequência. A primeira é o fato de não haver nenhuma crise convulsiva relatada em decorrência do seu uso. Ao contrário, este tipo de estimulação tem sido estudado como forma de tratamento da epilepsia ${ }^{17}$. Com a estimulação de alta frequência, por outro lado, sabemos que existe potencial para desencadeamento de crises convulsivas, com alguns relatos publicados ${ }^{18}$.

A outra vantagem é o custo. A depressão é doença de alta prevalência, atingindo todas as classes sociais. Segundo previsões, é possível que em 2020 seja a segunda doença que mais causará perda de anos de vida produtiva, com incidência apenas menor que as doenças isquêmicas do coração ${ }^{19}$. Neste contexto, a estimulação magnética transcraniana surge como instrumento neuropsiquiátrico de grande utilidade para este século ${ }^{20}$.

Sabemos da carência de recursos para os hospitais universitários e públicos no nosso país, com uma grande dificuldade para a aquisição de novos equipamentos. Sob esta ótica, é importante observar que o valor de um aparelho de estimulação magnética transcraniana de baixa frequência é bem menor do que o de alta, pois tal equipamento não necessita de sistema de refrigeração para a bobina.

Há, atualmente, grande variedade de protocolos utilizados nos trabalhos com estimulação magnética para o tratamento da depressão, em relação, principalmente, à frequência, porcentagem do limiar motor utilizado, número de sessões e número de pulsos. Este fato sugere que mais estudos são necessários para se chegar a um consenso quanto à metodologia ideal de aplicação deste tratamento.

$O$ estudo atual sugere que, para o presente protocolo de EMT de baixa frequência, o número de sessões deve ser superior a oito, na maioria dos casos. Também levanta a questão de possível correlação entre depressão com sintomas psicóticos e falta de resposta significativa à EMT, o que deve ser objeto de estudos posteriores.

\section{BIBLIOGRAFIA}

1. Barker AT, Jalinous R, Freeston IL. Noninvasive magnetic stimulation of human motor cortex. Lancet 1985;1:1106-1107.

2. Brasil-Neto JP, McShane LM, Fuhr P, Hallett M, Cohen LG. Topographic mapping of the human motor cortex with magnetic stimulation: factors affecting accuracy and reproductibility. Electroencephalogr Clin Neurophysiol 1992;85:9-16.

3. Pascual-Leone A, Tormos JM, Keenan J, Tarazona F, Canete C, Catala MD. Study and modulation of human cortical excitability with transcranial magnetic stimulation. J Clin Neurophysiol 1998;15:333-343.

4. Brasil-Neto JP, Cohen LG, Panizza M, Nilsson J, Roth BJ, Hallet M. Optimal focal transcranial magnetic activation of the human cortex: effects of coil orientation, shape of the induced current pulse, and stimulus intensity. J Clin Neurophysiol 1992;9:132-136.

5. Pascual-Leone A, Valls-Sole J, Brasil-Neto JP, Cohen LG, Hallet M. Akinesia in Parkinson's disease: I. Shortening of simple reaction time with focal, single-pulse transcranial magnetic stimulation. Neurology 1994;44:884-891.

6. Chen R, Classen J, Gerloff C, Wassermann EM, Hallett M, Cohen LG. Depression of cortex excitability by low-frquency transcranial magnetic stimulation. Neurology 1997;48:1398-1403.

7. George MS, Wassermann EM, Williams WA. Daily repetitive transcranial magnetic stimulation (rTMS) improves mood in depression. NeuroReport 1995;6:1853-1856.

8. Wassermann E, Lisanby $\mathrm{SH}$. Therapeutic application of repetitive transcranial magnetic stimulation: a review. Clin Neurophysiol 2001; 192:1367-1377.

9. Speer AM, Kimbrell TA, Wassermann EM, et al. Oppositive effects of high and low frequency rTMS on regional brain activity in depressed patients. Biol Psychiatry 2000;48:1133-1141.

10. Menkes DL, Bodnar P, Ballesteros RA, Swenson MR. Right frontal lobe slow frequency repetitive transcranial magnetic stimulation (SFr-TMS) is an effective treatment for depression: a case-control pilot study of safety and efficacy. J. Neurol. Neurosurg Psychiatry 1999;67:113-115.

11. Kaplan H, Sadock B, Greeb J. Compêndio de psiquiatria ciências do comportamento e psiquiatria clínica. 7.Ed. Batista D. Porto Alegre: Artes Médicas, 1997:493-544

12. Moreno RA, Moreno DH. Escalas de avaliação para depressão de Hamilton (HAM-D) e Montgomery-Asberg (MADRS). In Gorenstain C, Andrade LHSG, Zuardi AW (eds). Escalas de avaliação clínica em psiquiatria e psicofarmacologia. São Paulo: Lemos-Editorial, 2000:65-87.

13. Pascual-Leone A, Rubio B, Pallardó F, Catalá MD. Rapid-rate transcranial magnetic stimulation of left dorsolateral prefrontal cortex in drugresistant depression. Lancet 1996;347:233-237.

14. Nilsson J, Panizza M, Roth BJ, el al. Determing the site of stimulation during magnetic stimulation of a peripheral nerve. Eletroencephalogr Clin Neurophysiol 1992;85:253-264.

15. Geller V, Grisaru N, Abarbanel JM, Lemberg T, Belmaker RH. Slow magnetic stimulation of cortex prefrontal in depression and schizophrenia. Progr Neuropsycopharmacol Biol Psychiatry 1997;21:105-110.

16. Hoffman RE, Boutros NN, Berman RM, et al. Transcranial magnetic stimulation of left temporal cortex in three patients reporting hallucinated "voices". Biol. Psychiatry 1999;46:130-132.

17. Tassinari CA, Michelucci R, Forti A, et al. Transcranial magnetic stimulation in epileptic patients: usefulness and safety. Neurology 1990; 40:1132-1133.

18. Wassermann EM. Risk and safety of repetitive transcranial magnetic stimulation: report and suggested guidelines from the International Workshop on the Safety of Repetitive Transcranial Magnetic Stimulation. Eletroencephalogr Clin Neurophysiol 1998;108:1-16.

19. Murray CJ, Lopez AD. Alternative projections of mortality and disability by cause 1990-2020: Global Burden of Disease Study. Lancet 1997;349:1498-1504.

20. George MS, Wassermann EM, Post RM. Transcranial magnetic stimulation: a neuropshychiatric tool for the 21st century. J Neuropshychiatry Clin Neurosci 1996;8:373-382. 